\title{
SOME REMARKS ON BRAUER'S THIRD MAIN THEOREM
}

\author{
ARYE JUHÁSZ
}

\begin{abstract}
We consider two classes of $p$-blocks of a finite group $G$ which have the property that for every block $B$ of them and every subgroup $H$ of $G$, $H$ has only a small number of admissible blocks $b$ with $b^{G}=B$. In this they are similar to the principal block of $G$. These blocks are described by means of certain modules they contain.
\end{abstract}

Introduction. Let $G$ be a finite group of order $|G|$ and $\chi$ a complex character of $G$ in a $p$-block $B$ of $G, P|| G \mid$. If $u$ is an element of $G$ of order a power of $p$ and $r$ is an element of $G$ of order prime to $p$ which commutes with $u$, then by $[3,1.1]$

$$
\chi(u r)=\sum_{b \in \beta} \sum_{\phi \in[b]} d(\chi, \phi) \phi(r)
$$

where $\beta$ is the set of all the admissible blocks of $C_{G}(u)$ with $b^{G}=B, d(\chi, \phi)$ the generalized decomposition numbers and $[b]$ is a basic set for $b$. (See [3].) Here, following Brauer $[3,2 \mathrm{C}]$, we call a block $b$ of a subgroup $H$ of $G$ admissible if the centralizer of one of its defect groups is contained in $H$.

When using $(*)$, one has to have some information on $\beta$. The aim of this work is to supply sufficient conditions for $\beta$ to be "small". A typical result of this kind is Brauer's Third Main Theorem which states that if $B$ is the principal block of $G$ and $H$ is any subgroup of $G, b$ an admissible block of $H$, then $b^{G}=B$ if and only if $b$ is the principal block of $H$. For $H=C_{G}(u)$ this implies, of course, that $\beta$ in (*) consists only of the principal block of $H$. Later, Brauer showed in [2] that, in general, blocks which contain a linear representation have a similar property. We shall describe more cases like this in terms of modular representations:

Call a block $B$ of $K G$ a quasi-principal block if every subgroup of $G$ which has admissible blocks, has exactly one admissible block $b$ with $b^{G}=B$. Call $B$ a weakprincipal block if for every subgroup $H$ of $G$ which has admissible blocks and for every $p$-subgroup $Q$ of $H$ there is at most one admissible block $b$ of $H$ with $b^{G}=B$.

In these terms our main results are the following.

THEOREM 1. Let $B$ be a block of $K G$ and $S$ a Sylow p-subgroup of $G$. Assume that $K$ is a splitting field for the subgroups of $G$ and $B$ contains an indecomposable $K G$-module $M$ of $K$-dimension $d \leq p-1$. If $\left(d,\left|N_{G}(S) / S C_{G}(S)\right|\right)=1$ then $B$ is quasi-principal. Moreover, let $H$ be a subgroup of $G$ which has admissible blocks. Then $H$ has exactly one admissible block $b$ with $b^{G}=B$ and it contains all the components of $M_{H}$.

Received by the editors June 29, 1981 and in revised form, February 25, 1982.

1980 Mathematics Subject Classification. Primary $20 \mathrm{C20.}$

Key words and phrases. Block theory, modules in blocks, defect groups, vertices. 
THEOREM 2. Let $K$ be a splitting field for the subgroups of $G$ and let $B$ be a block of $K G$ with a defect group $D$. If $B$ contains an indecomposable $K G$-module $M$ such that all the components of $M_{D C_{G}(D)}$ belong to the same block $b$, then $b^{G}=B$, $D C_{G}(D)$ contains a Sylow p-subgroup of $G$ and $B$ is weak-principal. If $D$ is a Sylow p-subgroup of $G$ then $B$ is quasi-principal.

The proof of Theorem 2 provides a sufficient (and necessary) condition for a block $B$ of $K G$ to be the principal block (Theorem 3). As a corollary we get a result of Cassey and Gaschütz [5] concerning certain elementary abelian sections of $G$ of order a power of $p$.

In $\S 1$ we quote the necessary results and fix the notation. In $\S 2$ we investigate weak-principal blocks while in $\S 3$ we deal with quasi-principal blocks.

ACKNOWLEDGEMENTS. This work was written in part while I was a Rothchild Fellow and a Visiting Fellow at the University of Warwick. I am grateful to the Foundation for the grant and to the Mathematics Institute at the University of Warwick, especially to Professors J. A. Green, T. O. Hawkes and S. E. Stonehewer for their generous hospitality.

I am indebted to Professor Walter Feit for drawing my attention to [2]. I also wish to express my thanks to the referee for his useful suggestions.

1. Notation and preliminary results. In what follows fix the following notation: $G$ a finite group, $H$ a subgroup of $G, S$ a Sylow $p$-subgroup of $G, K$ a field of characteristic $p, p|| G \mid$, which is a splitting field for $G$ and its subgroups, $B$ a block of $K G$ with idempotent $E$ and $M$ an indecomposable $K G$-module in $B$. Write "component" for "indecomposable direct summand" and for every subgroup $X$ of $G$ and block $c$ of $X$ denote by $\left(c, M_{X}\right)$ the fact that $c$ contains a component of $M_{X}$. Other notation is standard, see [6].

We recall some definitions and results from Brauer's work [3]. Denote by $\underline{P}$ the set of all pairs $(Q, b)$, where $Q$ is a $p$-subgroup of $G$ and $b$ is a block of $Q C(Q)$, with the defect group $Q$. Here $C(Q)$ stands for $C_{G}(Q)$.

DEFINITION 1.1 $[3,3.1]$. Two pairs $\left(P, b^{*}\right)$ and $\left(Q, b^{* *}\right)$ of $P$ are linked if $Q$ is a normal subgroup of $P, Q \neq P$, and if $\left(b^{*}\right)^{P C(Q)}=\left(b^{* *}\right)^{P C(\bar{Q})}=b, b$ a block of $P C(Q)$.

LEMMA $1.2[3,3 \mathrm{~A}]$. If the pairs $\left(P, b^{*}\right)$ and $\left(Q, b^{* *}\right)$ of $\underline{P}$ are linked and $b=$ $b^{* P C(Q)}$, then

(a) $b$ and $b^{*}$ have the same defect group $P$.

(b) $b$ and $b^{* *}$ have the same corresponding central idempotent $e$.

(c) $C_{P}(Q) \subseteq Q$.

LEMMA 1.3. Let $B$ be a block of $K G$ with defect group $D$ and $\tilde{b}$ an admissible block of $H$ with a defect group $Q, Q \leq D$, such that $\tilde{b}^{G}=B$. Then

(a) $[3, \mathrm{~J}]$ There is a pair $(Q, b)$ in $\underline{P}$ with $b^{H}=\tilde{b}$ (hence $\left.b^{G}=B\right)$.

(b) $[3,3 \mathrm{E}, 3 \mathrm{~F}]$ If $\left(Q, b^{*}\right) \in \underline{P}$ and $b^{* G}$ has a defect group $D$ with $D \neq Q$, then there exists a p-subgroup $P$ of $G$ with $|P: Q|=p$ and a pair $\left(P, b^{* *}\right)$ in $\underline{P}$ such that $\left(Q, b^{*}\right)$ and $\left(P, b^{* *}\right)$ are linked.

Finally, we recall some results from [8]

PROPOSITION $1.4[8,3(\mathrm{a})]$. If $B$ has a defect group $D$ then every admissible block $b$ of $H$ which satisfies $b^{G}=B$ and has a defect group $D$ contains a component of $M_{H}$. 
Proposition 1.5 [8, Proposition]. Let $D$ be a $p$-subgroup of $G$ which is not normal in any Sylow p-subgroup of $G$ and let $H$ be a normal subgroup of $N_{G}(D)$ which contains $D C_{G}(D)$ and a Sylow p-subgroup of $N_{G}(D)$. If $B$ is a block of $K G$ with defect group $D$ and $M$ is an indecomposable $K G$-module in $B$, then $M_{H}$ has a component in a block $b$ of $K H$ with $b^{G} \neq B$, provided $K$ is a splitting field for $D C_{G}(D)$.

PROPOSITION 1.6 [10, 1.6(a)]. Let $\beta$ be the set of all the defect groups of $B$ and denote $D=\left\{D \cap H \mid D \in \beta, C_{G}(D \cap H) \subseteq H\right\}$. Let $D_{0}$ be the set of all the maximal elements of $D$ with respect to " $\leq_{H}$ ". Then

(a) If $b$ is an admissible block of $H$ with $b^{G}=B$ then $b$ has a defect group in $D$. $Q$.

(b) For every $Q \in D_{0}$ there exists a block $b$ of $H$ with $b^{G}=B$ having defect group

\section{Weak-principal blocks.}

LEMMA 2.1. Let $B$ be a block of $K G$ with a defect group $D$ and let $b$ be a olock of $D C(D)$ having defect group $D$ with $b^{G}=B$. (See Lemma 1.3(a).) Let $P$ be a Sylow $p$-subgroup of $N_{G}(D)$, and assume that $b$ is the only block of $D C(D)$ with $b^{G}=B$. Then

(a) $P=D C_{P}(D)$.

(b) $D$ is a Sylow p-subgroup of $G$ if and only if $Z(D)$ is a Sylow p-subgroup of $C_{G}(D)$.

In particular, if $B$ is weak-principal, then (a) and (b) hold.

ProOF. (a) By $[3,4 \mathrm{~K}]$ the set of all the blocks $b_{i}$ of $D C(D)$ with $b_{i}^{G}=B$ forms an $N_{G}(D)$-conjugacy class of blocks. Therefore, if $T(b)=\left\{x \in N_{G}(D) \mid x^{-1} b x=b\right\}$, then $\left|N_{G}(D): T(b)\right|=1$ by assumption, i.e. $N_{G}(D)=T(b)$. On the other hand, $D C_{P}(D)$ is a Sylow $p$-subgroup of $T(b)$, by [12]. Thus $P=D C_{P}(D)$.

(b) If $D$ is a Sylow $p$-subgroup of $G$ then certainly $Z(D)$ is a Sylow $p$-subgroup of $C_{G}(D)$.

If $Z(D)$ is a Sylow $p$-subgroup of $C_{G}(D)$ then $P=D$ by part (a), hence $D$ is the Sylow $p$-subgroup of $N_{G}(D)$. Therefore $D$ is a Sylow $p$-subgroup of $G$.

LEMMA 2.2. Let $D$ be a defect group of $B$ and set $H_{0}=D C(D)$. If all the components of $M_{H_{0}}$ belong to the same block $b$ of $H_{0}$, then

(a) $b^{G}=B$.

(b) $D C_{G}(D)$ contains a Sylow p-subgroup of $G$.

(c) For every subgroup $Q$ of $D$ there is at most one block $\tilde{b}$ of $Q C(Q)$ having defect group $Q$ and satisfying $\tilde{b}^{G}=B . \tilde{b}$ contains all the components of $M_{Q C(Q)}$.

ProOF. (a) Follows by Proposition 1.4.

(b) Follows by Lemma 2.1(a), Propositions 1.4 and 1.5.

(c) By induction on $|D: Q|$. For $|D: Q|=1$ this is just the assumption, by Proposition 1.4. Let $Q$ be a proper subgroup of $D$ and assume that $\tilde{b}$ is a block of $Q C(Q)$ with $\tilde{b}^{G}=B$ having defect group $Q$. Then by Lemma $1.3(\mathrm{~b})$ there is a subgroup $P$ of $D$ containing $Q$ such that $|P: Q|=p$ and a block $b^{* *}$ of $P C(P)$ such that $(Q, \tilde{b})$ and $\left(P, \tilde{b}^{* *}\right)$ are linked. By Lemma $1.2(\mathrm{~b}) b^{* * G}=B$ and by the induction hypothesis $b^{* *}$ contains all the components of $M_{P C(P)}$. Let $L$ be a component of $M_{P C(Q)}$. By the induction hypothesis $b^{* *}$ contains all the components of $L_{P C(P)}$, 
as $L \mid M_{P C(Q)}$. Since $b$ and $b^{* *}$ have the same defect group $P$ by Lemma 1.2(a), $L$ must belong to $b$ by Proposition 1.4. Consequently $M_{P C(Q)}$ belongs to $\tilde{b}$, hence $M_{Q C(Q)}$ belong to $b$ by Lemma $1.2(\mathrm{~b})$, since $M e=M, e$ as in Lemma 1.2(b). This obviously proves part (c).

Proposition 2.3. Let $B$ be a block of $K G$ with a defect group $D$. If $D$ is a Sylow p-subgroup of $G$ then $B$ is quasi-principal if and only if $B$ is weak-principal.

Proof. Let $H=\left\{H \leq G \mid H\right.$ has admissible blocks $b$ with $\left.b^{G}=B\right\}$. If $B$ is quasi-principal then it is weak-principal, by definition. Let $B$ be a weak-principal block of $K G$. Let $H \in \mathcal{H}$ and assume that $b$ is an admissible block of $K H$ with $b^{G}=B$, having a defect group $Q$. Let $S$ and $P$ be Sylow $p$-subgroups of $G$ and $H$ respectively with $Q \leq P \leq S$. We show by induction on $|S: Q|$ that $Q=P$. Then the result follows, since $B$ is weak-principal. For $S=Q$ this is obvious. Assume that $|S: Q|>1$ and the result holds for every subgroup $H^{*}$ of $G$ and admissible block $b^{*}$ of $H^{*}$ with $b^{* G}=B$ having defect group $Q^{*} \gtreqless Q$. By assumption $B$ has maximal defect, hence by Proposition $1.6 K H$ has a block $\tilde{b}$ with $\tilde{b}^{G}=B$ having defect group $P$. Since $Q \leq P$, it follows by Bauer's First Main Theorem that $Q C(Q)$ has blocks $\beta$ and $\tilde{\beta}$ with $\beta^{H}=b$ and $\tilde{\beta}^{H}=\tilde{b}$, respectively. Let $H^{*}=N_{G}(Q)$. Then $b^{*}=\beta^{H^{*}}$ and $\tilde{b}^{* *}=\tilde{\beta}^{H^{*}}$ have defect groups $\gtreqless Q$ by Brauer's First Main Theorem, hence $\tilde{\beta}^{H^{*}}=\beta^{H^{*}}$ by the induction hypothesis since $B$ is weak-principal and $\tilde{\beta}^{G}=$ $\beta^{G}=B$. So $\beta$ and $\tilde{\beta}$ are conjugate in $H^{*}$ hence both have defect group $Q$. But then $\beta=\tilde{\beta}$, since $B$ is weak-principal and $\tilde{b}=\tilde{\beta}^{H}=\beta^{H}=b$. Hence $Q=P$, and $\tilde{b}$ is the only admissible block of $H$ with $\tilde{b}^{G}=B$.

We now prove the main result of this section.

THEOREM 2. Let $K$ be a splitting field for the subgroups of $G$ and let $B$ be a block of $K G$ with a defect group $D$. If $B$ contains an indecomposable $K G$-module $M$ such that all the components of $M_{D C_{G}}(D)$ belong to the same block $b$, then $b^{G}=B$, $D C_{G}(D)$ contains a Sylow p-subgroup of $G$ and $B$ is weak-principal. If $D$ is a Sylow $p$-subgroup of $G$ then $B$ is quasi-principal.

Proof. $b^{G}=B$ by Lemma $2.2(\mathrm{a}), D C_{G}(D)$ contains a Sylow $p$-subgroup of $G$ by Lemma $2.2(\mathrm{~b})$ and $B$ is weak-principal by Lemma $2.2(\mathrm{c})$. If $D$ is a Sylow $p$-subgroup of $G$ then $B$ is quasi-principal by Proposition 2.3.

COROLLARY 1. Let $K$ be a splitting field for the subgroups of $G, B$ a block of $K G, M$ an indecomposable $K G$-module in $B$ and $S$ a Sylow p-subgroup of $G$. If all the components of $M_{S C(S)}$ belong to the same block then each of the following conditions on $M$ implies that $B$ is quasi-principal:

(a) $S$ is contained in the kernel of the representation afforded by $M$.

(b) $M$ has $K$-dimension prime to $p$.

Proof. In case (a) $M$ has vertex $S$ by $[6,53.8]$ and in case (b) $M$ has vertex $S$ by $[6,52.4]$. So in both cases $B$ has defect group $S$. Consequently $B$ is quasi-principal hy Theorem 2 .

The proof of Theorem 2 yields

THEOREM 3. Let $B$ be a block of $K G$ and $M$ an indecomposable $K G$-module in $B$. Let $D$ be a defect group of $B$. Then each one of the following conditions implies that $B$ is the principal block of $K G$. 
(a) $C_{G}(D)$ is contained in the kernel of the representation afforded by $M$.

(b) $C_{G}(D)$ has a normal $p$-complement $T$ and $T$ is contained in the kernel of the representation afforded by $M$.

Proof. Note that in Theorem 4 there is no restriction on the field $K$.

(a) Assume first that $K$ is a splitting field for the subgroups of $G$. Since $C_{G}(D)$ acts trivally on $M$ by assumption, $M_{C(D)}$ is the direct sum of trivial one-dimensional $K C(D)$ modules, hence $M_{C(D)}$ belongs to the principal block of $K C(D)$. On the other hand, it is elemenary and well known (see e.g. [1, 2.9(1)]) that every central primitive idempotent of $D C(D)$ lies in $C(D)$ and every central primitive idempotent of $C(D)$ is a central idempotent of $D C(D)$, as $D$ centralizes $C(D)$. Consequently, the principal blocks of $C(D)$ and $D C(D)$ have the same idempotent $e$ and so $M_{D C(D)}$ belongs to the principal block of $D C(D)$, as $M e=M$. Hence by Theorem 3 and Brauer's Third Main Theorem (or Theorem 1) $M$ belongs to the principal block of $K G$.

Assume now the general case and let $K \subseteq \bar{K}$ be a splitting field for the subgroup of $G$. Let $M \otimes \bar{K}=L_{1} \oplus \cdots \oplus L_{r}, L_{i}$ indecomposable $\bar{K} G$-modules. Since $C_{G}(D)$ acts trivially on the $L_{i}, 1 \leq i \leq r, M \otimes \bar{K}$ belong to the principal block of $\bar{K} G$, by the above. Assume $E \otimes 1=e_{1}+\cdots+e_{t}, e_{i}$ central primitive idempotents of $\bar{K} G$. Then $(M \otimes 1)(E \otimes 1)=M \otimes 1$ implies that $(M \otimes 1) e_{i} \neq 0$ for some $i, 1 \leq i \leq t$. Therefore $e_{i}$ is the idempotent of the principal block of $\bar{K} G$ and moreover $(M \otimes \bar{K}) e_{i}=M \otimes \bar{K}$, by the above. We want to show that $E$ is the principal block of $K G$. Let $L$ be the trivial representation of $K G$. Then $L \otimes \bar{K}$ is the trivial representation of $\bar{K} G$; hence $(L \otimes \bar{K}) e_{i}=L \otimes \bar{K}$. Consequently, $(L \otimes \bar{K})(E \otimes 1)=L \otimes \bar{K}$. This easily implies $L E=L$, i.e. $M$ belongs to the principal block of $K G$.

(b) By [4 or 11] every idempotent of $D C(D)$ lies in $T$. Therefore, as in part (a) $M_{D C(D)}$ belongs to the principal block of $D C(D)$. The rest is the same as in part (a).

COROLLARY 2 [5]. Let $R_{1}$ and $R_{2}$ be two normal p-subgroups of $G$ such that $R_{1} \subseteq R_{2}$ and $R_{2} / R_{1}$ is elementary abelian. Then the $\mathbf{Z}_{P} G$-module $R_{2} / R_{1}$ belongs to the principal block.

Proof. Assume first that $R_{2} / R_{1}$ is indecomposable and belongs to $B$ with defect group $D$. Then $R_{2} \leq D$ by $[3,2]$. Therefore $C(D) \leq C\left(R_{2}\right)$, hence $C(D)$ is contained in the kernel of the representation afforded by $R_{2} / R_{1}$. Consequently $R_{2} / R_{1}$ belongs to the principal block of $K G$, by Theorem $3(\mathrm{a})$. If $R_{2} / R_{1}$ decomposes, then the same argument for each component of it shows that $R_{2} / R_{1}$ belongs to the principal block.

COROLLARY 3. Let $S$ be a Sylow p-subgroup of $G$ and $T$ a normal p-complement of $C_{G}(S)$. Then the principal block of $G$ contains at least $\left|G: T G^{\prime}\right|$ linear characters.

Proof. Every linear character of $G$ which contains $G^{\prime} T$ in its kernel belongs to the principal block of $K G$, by Theorem 3(b). Hence the result follows.

COROLlARY 4. Let $D$ be a normal p-subgroup of $G$. Then the KG-module $K\left(G / C_{G}(D)\right)$ belongs to the principal block of $K G$.

Proof. Every component of $K\left(G / C_{G}(D)\right)$ contains $C_{G}(D)$ in its kernel. Since $D$ is a normal $p$-subgroup of $G$, every block of $K G$ has defect group containing $D$. Consequently, the centralizer of every defect group is contained in the kernel of every component of $K\left(G / C_{G}(D)\right)$, hence the result follows by Theorem 3(a). 


\section{Quasi-principal blocks.}

LEMMA 3.1. Let $B$ be a block of $K G$ with a defect group $D$ and $b$ an admissible block of $K H$. Assume that $B$ has an indecomposable $K G$-module $M$ which satisfies

(**) Every component of $M_{D^{*} C\left(D^{*}\right)}$ has vertex $\geq D^{*}$, for every $D^{*}<D$.

Then $b^{G}=B$ if and only if $b$ contains a component of $M_{H}$

PROOF. Let $L_{1}, \ldots, L_{t}$ be all the components of $M_{H}$ and let $b_{i}$ be the block of $H$ which contains $L_{i}, b_{i}$ with defect group $D_{i}, 1 \leq i \leq t$. Assume that $b$ has defect group $D_{0} \leq D$. Then we claim

(1) $b_{i}^{G}=B$ for $1 \leq i \leq t$. (In other words $\left(b, M_{H}\right)$ implies $b^{G}=B$.)

(2) If $D_{0} \triangleleft G, H=D_{0} C\left(D_{0}\right)$ and $b^{G}=B$ then $b=b^{i}$ for some $i, 1 \leq i \leq t$.

(3) Let $\beta$ be a block of $D_{0} C\left(D_{0}\right)$ with $\beta^{H}=b$. Then $\left(\beta^{N_{G}\left(D_{0}\right)}, M_{N_{G}\left(D_{0}\right)}\right)$ implies $b=b_{i}$ for some $i, 1 \leq i \leq t$, (i.e. $\left(b, M_{H}\right)$ ).

Assume we have proved claims (1)-(3). Then in view of (1) it remains only to show that $b^{G}=B$ implies $\left(b, M_{H}\right)$. Thus assume $b^{G}=B$ and prove $\left(b, M_{H}\right)$ by induction on $\left|D: D_{0}\right|$.

If $D=D_{0}$, then our assertion is Proposition 1.4.

Therefore assume $D_{0} \neq D$ and suppose we have proved the assertion for every subgroup $H^{\prime}$ and admissible block $b^{\prime}$ of $H$ with defect group $D_{0}^{\prime}>D_{0}$ and prove for $H$ and $b$. Since $H$ is arbitrary, this will do. Let $\beta$ be as in (3). Then by Brauer's First Main Theorem $\beta^{N_{G}\left(D_{0}\right)}$ has a defect group $D^{\prime}$, strictly containing $D_{0}$, as $D_{0} \neq D$. Therefore $N_{G}\left(D_{0}\right)$ satisfies the induction hypothesis with $N_{G}\left(D_{0}\right)$ in place of $H^{\prime}$ and $\beta^{N_{G}\left(D_{0}\right)}$ in place of $b^{\prime}$, hence $\left(\beta^{N_{G}\left(D_{0}\right)}, M_{N_{G}\left(D_{0}\right)}\right)$. By (3) this proves the lemma. Now we prove claims (1)-(3).

(1) Since every component of $M_{D_{i} C\left(D_{i}\right)}$ has vertex $\geq D_{i}$, obviously every $L_{i}$ has vertex $V_{i} \geq D_{i}$, hence $C\left(V_{i}\right) \leq C\left(D_{i}\right)$ and $b^{G}=B$ by Nagao's theorem [6, 56.5].

(2) All block $b^{\prime}$ of $H$ with $b^{\prime G}=B$ are conjugate in $G$. Since every such $b^{\prime}$ contains a component of $M_{H}$, every such $b^{\prime}$ is one of the $b_{i}, 1 \leq i \leq t$.

(3) By (2) $\left(\beta, M_{D_{0} C\left(D_{0}\right)}\right)$. Therefore $\left(b, M_{H}\right)$ by (1), as $b=\beta^{H}$. Here we used the fact that if $M$ satisfies (**) then every component of $M_{H}$ satisfies it.

We come to the main result of this section:

THEOREM 1. Let $B$ be a block of $K G$ and $S$ a Sylow p-subgroup of $G$. Assume that $K$ is a splitting field for the subgroups of $G$ and $B$ contains an indecomposable $K G$-module $M$ of $K$-dimension $d \leq p-1$. If $\left(d,\left|N_{G}(S) / S C_{G}(S)\right|\right)=1$ then $B$ is quasi-principal. Moreover, let $H$ be a subgroup of $G$ which has admissible blocks. Then $H$ has exactly one admissible block $b$ with $b^{G}=B$ and it contains all the componets of $M_{H}$.

Proof. Since $\operatorname{dim}_{K} M \leq p-1, M$ and all the components of $M_{H}$ have vertex a Sylow $p$-subgroup of $G$ and $H$ respectively, by $[7,22.6]$. Therefore, every block $b$ of $K H$ with $b^{G}=B$ contains a component of $M_{H}$ by Lemma 3.1 and has maximal defect by $[6,54.10]$. It suffices to show that $B$ is weakly-principal. To this end, we may assume $H=S C(S), S$ a Sylow $p$-subgroup of $G$, by Theorem 2 .

Let $b_{1}, \ldots, b_{r}$ be all the block of $H$ with $b_{i}^{G}=B$. Then by Lemma $3.1 \operatorname{dim}_{K} M=$ $\sum_{i=1}^{r} \operatorname{dim}_{K} M e_{i}$ where $e_{i}$ is the central idempotent of $b_{i}, 1 \leq i \leq r$. Since $\operatorname{dim}_{K} M e_{i}$ $=\operatorname{dim}_{K} M x^{-1} e_{i} x$ for every $i, 1 \leq i \leq r$, and every $x \in N_{G}(S)$ and since the $b_{i}$, $1 \leq i \leq r$, form a $N_{G}(S)$ conjugacy class of blocks of $H, d=\operatorname{dim}_{K} M=r \operatorname{dim} M e_{i}$. Hence $r \mid d$. On the other hand, $r=\left|N_{G}(S): T\left(b_{1}\right)\right|$ hence $r \| N_{G}(S): S C(S) \mid$. 
Consequently, $r \mid\left(\left|N_{G}(S): S C(S)\right|, d\right)=1$ and the result follows by Theorem 2 . Here $T\left(b_{1}\right)=\left\{g \in N_{G}(S) \mid g^{-1} b_{1} g=b_{1}\right\}$.

The following corollaries are immediate from Theorem 1.

COROLLARY $5[2,4 \mathrm{E}, 4 \mathrm{D}]$. If $B$ contains a linear representation then $B$ is quasiprincipal.

CoRollary 6. If $N_{G}(S)=S C_{G}(S)$, then every block $B$ which contains a representation of degree $\leq p-1$ is quasi-principal.

REMARKS. 1. Lemma 3.1 provides a short module-theoretic proof to Brauer's Third Main Theorem and to Corollary 1. This proof uses Brauer's First Main Theorem and Nagao's theorem and does not require that $K$ is a splitting field for the subgroups of $G[9]$.

2. All the results quoted from [3] have short module-theoretic proofs [10].

3. Theorem 3 for the special case $\operatorname{dim} M=1$ was proved in $[2,4 \mathrm{E}]$. Also it was proved there that the exact number of linear characters in the principal block is $\left|G: T G^{\prime}\right|$.

\section{REFERENCES}

1. J. L. Alperin and Michel Broué, Local methods in block theory, Ann. of Math. 110 (1973), 143-157.

2. R. Brauer, Some applications of the theory of blocks of characters of finite groups. IV, J. Algebra 17 (1971), 489-521.

3. - On the structures of blocks of characters of finite groups, (Proc. Second Internat. Conf. on the Theory of Groups), Lecture Notes in Math., vol. 372, Springer-Verlag, Berlin and New York, 1974.

4. R. Brauer and C. Nesbitt, On the modular representations of finite groups, Univ. of Toronto Studies Math. Ser. No. 4, 1937.

5. J. Cassey and W. Gaschutz, a note on blocks, (Proc. Second Internat. Conf. on the Theory of Groups), Lectures Notes in Math., vol. 372, Springer-Verlag, Berlin and New York, 1974.

6. L. Dornhoff, Groups representation theory, Parts A and B, Pure and Appl. Math., 7, Marcel Dekker, New York, 1972.

7. J. A. Green, Blocks of modular representations, Math. Z. 79 (1962), 100-115.

8. A. Juhász, Variations to a theorem of $H$. Nagao, J. Algebra 70 (1981), 173-178.

9. _ An elementary proof to two theorems of R. Brauer (unpublished).

10. _ On the distribution of restricted modules into blocks (submitted).

11. Y. Kawada, On blocks of group-algebras of finite groups, Sci. Rep. Tokyo Kyoiku Kaigaku Sect. A 9 (1966), 87-110.

12. D. Passman, Blocks and normal subgroups, J. Algebra 12 (1969), 569-575.

Department of Mathematics, Hebrew University, Jerusalem, IsRael 